\title{
Retroperitoneal Actinomycosis Mimicking a Malignant Neoplasm of Adrenal Gland
}

\author{
Mutlu Deger ${ }^{\mathrm{a}, \mathrm{d}}$, Fatih Gokalp ${ }^{\mathrm{a}}$, Behice Kurtaran ${ }^{\mathrm{b}}$, \\ Ilhan Tuncerc ${ }^{\mathrm{c}}$ Zuhtu Tansug ${ }^{\mathrm{a}}$
}

\begin{abstract}
Actinomycosis is a chronic disease characterized by abscess formation, tissue fibrosis, and draining sinuses. In this study, we aimed to present the clinical and radiological findings of a rare pathology, retroperitoneal actinomycosis case mimicking adrenal tumor and review the literature. Physicians must be aware of typical clinical presentations (such as cervicofacial actinomycosis following dental focus of infection, pelvic actinomycosis in women with an intrauterine device, and pulmonary actinomycosis in smokers with poor dental hygiene), but also that actinomycosis may mimic the malignancy process in various anatomical sites. Actinomycosis manifests as tumor-like lesions mainly in the cranio-cervical region $(60-80 \%)$, thorax (10$15 \%$ ), abdomen (5-20\%) and exceptionally in retroperitoneal organs. Positron emission tomography (PET) revealed hypermetabolic mass lesion $63 \times 52 \times 73 \mathrm{~mm}$ in size on the left adrenal gland showed a standardized uptake value (SUV) of 18.2. The patient underwent left adrenalectomy. The pathologic examination revealed actinomycosis. The patient was treated with antibiotics.
\end{abstract}

Keywords: Adrenal gland; Retroperitoneum; Actinomycosis

\section{Introduction}

Actinomycosis is a chronic disease characterized by abscess formation, tissue fibrosis, and draining sinuses. The causative organisms are non-motile, non-spore forming, non-acid fast and Gram-positive, pleomorphic, anerobic to microaerophilic filamentous bacterial rods [1]. The cervicofacial, thoracic, and

\footnotetext{
Manuscript accepted for publication August 19, 2015

aDepartment of Urology, Faculty of Medicine, University of Cukurova, Adana, Turkey

bepartment of Clinical Microbiology and Infectious Diseases, Faculty of Medicine, University of Cukurova, Adana, Turkey

'Private Tuncer Pathology Laboratory, Adana, Turkey

${ }^{\mathrm{d} C}$ Corresponding Author: Mutlu Deger, Department of Urology, Faculty of Medicine, University of Cukurova, 01330, Adana, Turkey.

Email: drmutludeger@gmail.com
}

doi: http://dx.doi.org/10.14740/jmc2282w abdominopelvic regions and the central nervous system (CNS) are most commonly involved in actinomycosis. Actinomycosis usually occurs in immunocompetent persons but may occur in persons with diminished host defenses. The portal of entry of Actinomyces species is typically a break in the mucosa of the gastrointestinal tract, anywhere from the mouth to the rectum; such a break may occur as a result of a dental procedure, bacterial suppuration, diverticulitis, appendicitis, surgery, or trauma $[2,3]$. Actinomycosis manifests as tumor-like lesions mainly in the cranio-cervical region (60-80\%), thorax (10-15\%), abdomen $(5-20 \%)$ and exceptionally in retroperitoneal organs [4].

In this study, we aimed to present the literature of clinical and radiological findings of a rare pathology, retroperitoneal actinomycosis case mimicking adrenal tumor.

\section{Case Report}

A 64-year-old man presented a 2-month history of general fatigue, a weight loss of $8-10 \mathrm{~kg}$ and left flank pain. He had no significant past medical or surgical history. A physical examination revealed no signs.

A soft tissue mass enhancing heterogeneous contrast on the left adrenal gland measuring $54 \times 54 \mathrm{~mm}$ was visualized and left renal vein depleted to anterior and superior by mass on the abdominal CT scans. Because of the tumor size, we performed an 18F-2-fluoro-deoxy-D-glucose-positron emission tomography (PET) scan to diagnose the malignant lesion. Hypermetabolic mass lesion $63 \times 52 \times 73 \mathrm{~mm}$ in size on the left adrenal gland showed a standardized uptake value (SUV) of 18.2 (Fig. 1). Endocrinological examinations including serum electrolytes, cortisol, urinary metanephrine, normetanephrine, adrenalin, noradrenalin and vanillyl mandelic acid, as well as plasma renin and aldosterone were within normal range. Due to the increased risk of malignancy for adrenal tumors larger than $4.0 \mathrm{~cm}$, he underwent a left adrenalectomy with no related complications. Macroscopic examination of retroperitoneal excisional material showed $5 \times 5 \mathrm{~cm}$ in size, $155 \mathrm{~g}$ in weight mass. In serial sections, pinkish, yellow, gray and green in color, solid, focal cystic formation with abscess was noticed. In microscopic examination, fibrous, hyalinized, adipose tissue and focal atrophic adrenal tissue infiltrated with dens neutrophile and eosinophile, leucocytes, lymphocytes, 

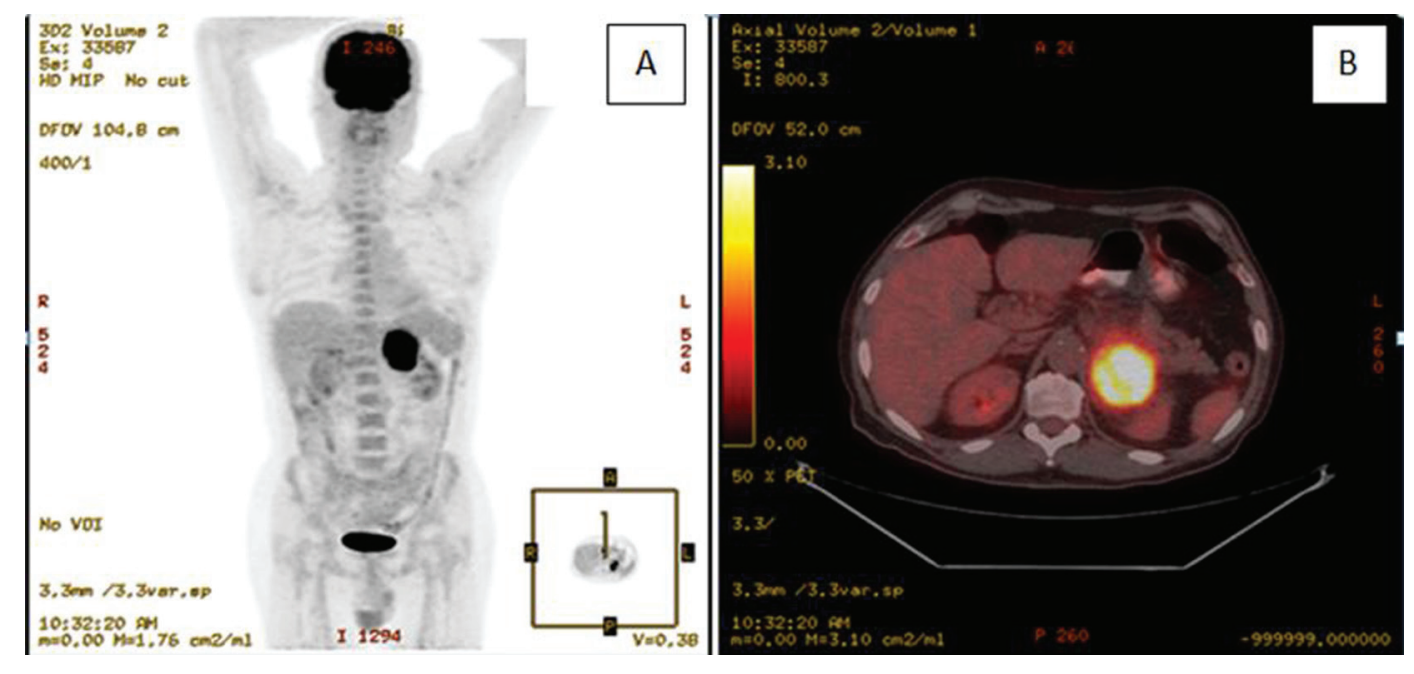

Figure 1. (A) Preoperative PET-CT scan. (B) Hypermetabolic mass lesion $63 \times 52 \times 73 \mathrm{~mm}$ in size on the left adrenal gland showed a standardized uptake value (SUV) of 18.2 .

plasmocytes and histiocytes (Fig. 2). Actinomyces colonies surrounded with leucocytes and abscess formations were seen.

Intravenous $1 \mathrm{~g}$ ampicilline was administered qid according to pathology after surgery. The patient stated that his weakness improved in the follow-up evaluation after 2 weeks. After completing parenteral ampicillin treatment, the patient returned to daily activities and continued with oral ampicillin treatment. The treatment is ongoing, and it will be continued for a period of 6 months.

\section{Discussion}

Actinomycosis is a rare chronic disease caused by Actinomyces israelii first described by J. Israel in 1878. It is a Gram-positive anerobic or microaerophilic filamentous bacteria that are non-spore forming [5]. Actinomycosis usually occurs in immunocompetent persons but may occur in persons with diminished host defenses. The port of entry of Actinomyces species is typically a break in the mucosa of the gastrointestinal tract, anywhere from the mouth to the rectum and such a break may occur as a result of a dental procedure, bacterial suppuration, diverticulitis, appendicitis, surgery, or trauma [2, 3]. In tissues, infecting Actinomyces species grow in microscopic or macroscopic clusters of tangled filaments that are surrounded by polymorphonuclear neutrophils. Subacute or chronic inflammation with granulation tissue, extensive fibrosis, and sinus tracts is present in the surrounding tissues, but giant cells and caseation necrosis are generally not seen. When grossly visible, clusters exude from soft tissues through sinus tracts, are pale yellow in color, and are called sulfur granules [6].

The MRI or CT features, however, resembled an abscess formation or a centrally necrotizing tumor. In our case, the initial misleading strong positive MIBG scintigraphic uptake was a false-positive finding with the clinical and finally histological findings. The uptake was probably caused by inflammatory involvement of the left adrenal gland with still unknown uptake mechanism [7]. In addition to antibiotics, the treatment
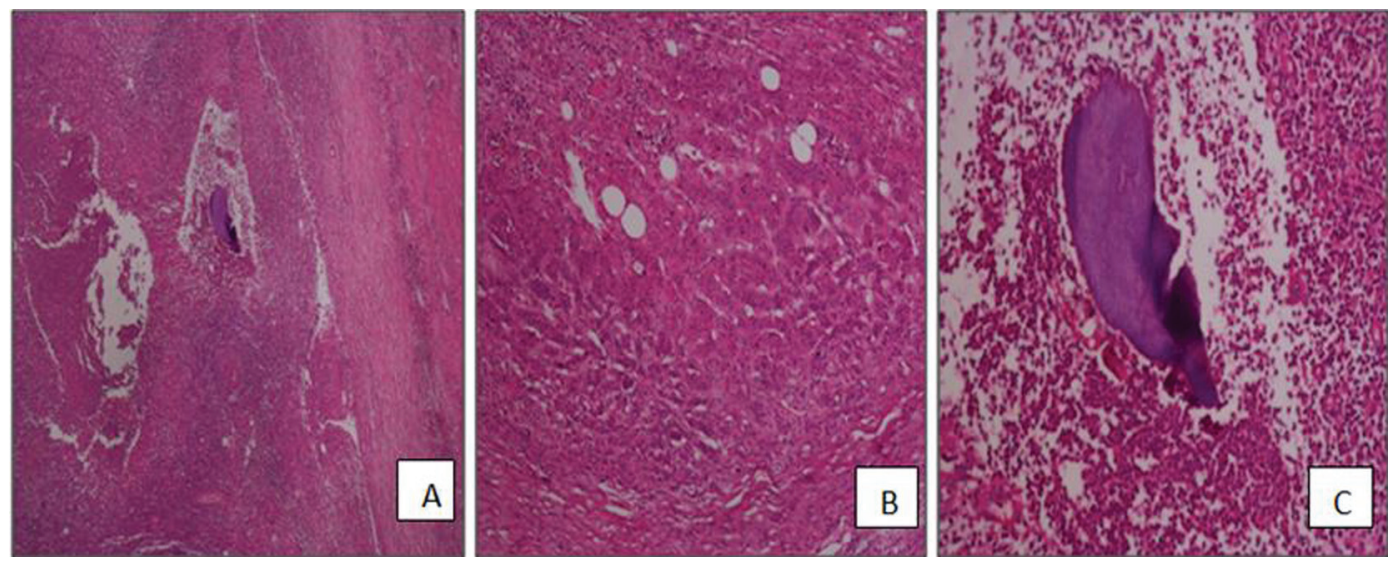

Figure 2. (A) Micrograph showing actinomyces colonies surrounded with neutrophile and eosinophile, leucocytes. Abscess formations were seen $(H \& E, \times 40)$. (B) High power of actinomyces colonies and inflammatory cells $(H \& E, \times 200)$. (C) Atrophic adrenal glands tissues in the fibrous collagen tissue with chronic inflammatory cells were seen (H\&E, $\times 100)$. 
of choice for retroperitoneal actinomycosis is interventional drainage of the abscess. After improving the patient's condition, no further interventional treatment was indicated. The use of penicillin, which is the drug of choice, has not been standardized. Since a preoperative diagnosis is only infrequently reported [2], all exudates or excised tissue should be cultured promptly under anerobic conditions to include the possibility of an actinomycosis abscess, as well as tuberculosis. The case reported shows that, even with misleading diagnostic results, an interventional puncture enables a quick and safe approach to both, verification of therapy and drainage of the abscess.

Abdominopelvic actinomycosis may be the most indolent and latent of all the clinical forms of actinomycosis and diagnosis may be delayed for months after the inciting event. One of the important radiologic characteristics of abdominopelvic actinomycosis is the aggressive nature of the infiltration. This disease's infiltrative nature, and its tendency to invade normal anatomic barriers, was confirmed in our patient. Such a pattern may be the result of proteolytic enzymes produced by Actinomyces. This results in extensive inflammatory fat infiltration with abscesses formation in the abdominal wall [8].

Actinomycosis is a rare chronic disease caused by $\mathrm{Ac}$ tinomyces spp., anerobic Gram-positive bacteria that normally colonize the human mouth and digestive and genital tracts. Physicians have to be aware of typical clinical presentations (such as cervicofacial actinomycosis following dental focus of infection, pelvic actinomycosis in women with an intrauterine device, and pulmonary actinomycosis in smokers with poor dental hygiene), but also must be aware that actinomycosis may mimic the malignancy process in various anatomical sites. Bacterial cultures and pathology are the cornerstones of diagnosis and require particular attention to prevent misdiagnosis. Patients with actinomycosis require prolonged (6 - 12 months) high doses of penicillin $\mathrm{G}$, ampicillin or amoxicillin, but the duration of antimicrobial therapy could likely be reduced ( 3 months) for patients in whom optimal surgical resection of infected tissues has been performed. Specific preventive measures (reduction of alcohol abuse, dental hygiene, change of intrauterine device every 5 years) may limit the occurrence of actinomycosis [9].

\section{References}

1. de Montpreville VT, Nashashibi N, Dulmet EM. Actinomycosis and other bronchopulmonary infections with bacterial granules. Ann Diagn Pathol. 1999;3(2):67-74.

2. Weese WC, Smith IM. A study of 57 cases of actinomycosis over a 36-year period. A diagnostic 'failure' with good prognosis after treatment. Arch Intern Med. 1975;135(12): 1562-1568.

3. Harvey JC, Cantrell JR, Fisher AM. Actinomycosis: its recognition and treatment. Ann Intern Med. 1957;46(5):868-885

4. Khalaff H, Srigley JR, Klotz LH. Recognition of renal actinomycosis: nephrectomy can be avoided. Report of a case. Can J Surg. 1995;38(1):77-79.

5. Berardi RS. Abdominal actinomycosis. Surg Gynecol Obstet. 1979;149(2):257-266.

6. Russo TA. Agents of actinomycosis. In: Mandell GL, Bennett JE, Dolin R, eds. Mandell, Douglas and Bennett's principles and practice of infectious diseases. New York: Churchhill Livingstone, 1995:2280-2288.

7. Jacobs A, Lenoir P, Delree M, Ramet J, Piepsz A. Unusual Tc-99m MDP and I-123 MIBG images in focal pyelonephritis. Clin Nucl Med. 1990;15(11):821-824.

8. Triantopoulou C, der Molen AV, Es AC, Giannila M. Abdominopelvic actinomycosis: spectrum of imaging findings and common mimickers. Acta Radiol Short Rep. 2014;3(2):2047981614524570.

9. Valour F, Senechal A, Dupieux C, Karsenty J, Lustig S, Breton P, Gleizal A, et al. Actinomycosis: etiology, clinical features, diagnosis, treatment, and management. Infect Drug Resist. 2014;7:183-197. 